\title{
Applying Benzene Tetracarboxylic Acid as a Linker in the Synthesis a Porous Ba (II)-Based MOF by Ultrasonic Method ${ }^{+}$
}

\author{
Targol Rahimi Masale Nezhad and Faranak Manteghi * \\ Department of chemistry, Iran University of Science and Technology, 1684613114, Tehran, Iran; \\ targol.rmn@gmail.com \\ * Correspondence: f_manteghi@iust.ac.ir \\ † Presented at the 23rd International Electronic Conference on Synthetic Organic Chemistry, 15 November- \\ 15 December 2019; Available online: https://ecsoc-23.sciforum.net/.
}

Published: 14 November 2019

\begin{abstract}
Metal-organic frameworks (MOFs), which are porous materials that are prepared from metal ion/clusters and multidentate organic ligands, have evolved to be next generation utility materials because of their usability in diverse applications. MOFs, as a class of interesting materials, have attracted great attention due to their controllable pore size and elaborately designed pore structure. In the past two decades, MOFs have exhibited versatile potential applications such as gas storage, gas separation, heterocatalysis, sensors, and luminescence. Most research works so far have focused on MOFs based on transition metal ions or rare-earth ions. In this review, a porous Ba (II)based MOF was synthesized with an ultrasonic method by using benzene-1,2,4,5-tetracaboxylic acid as an organic linker; this was characterized by X-Ray diffraction, Fourier transform infrared spectroscopy and scanning electron microscopy methods.
\end{abstract}

Keywords: metal-organic framework; barium ion; benzene-1,2,4,5-tetracaboxylic acid

\section{Introduction}

Metal-organic frameworks (MOFs) are inorganic-organic hybrid porous materials created by organic linkers and metal clusters, and they have attracted much attention as a growing class of porous materials and have evolved to be next generation utility materials. They have exhibited versatile potential applications such as gas storage, gas separation, heterocatalysis, sensors, and luminescence. Amongst the various MOFs, the main group alkaline earth metals is receiving increasing attention recently [1-4]. The unique characteristics of MOFs include a high surface area, a good thermal stability, uniform structured nanoscale cavities, a uniform but tunable pore size, controllable particle dimensions and morphologies, accessible cages and tunnels, specific adsorption affinities, and the availability of in-pore functionality and outer surface modifications [5]. One of the exceptional properties of MOFs relies on their ability to adapt their pore openings to accommodate guest species, and different modes of flexibility have been described. This "breathing" effect and swelling can produce a dramatic increase or decrease in cell volume without a loss of crystallinity or bond breaking [6]. These materials (MOFs) have shown great potential in gas adsorption, gas separation, catalysis, lithium storage [7], drug delivery [4] and biomedical imaging [5]. Among many kinds, alkali earth metal-organic frameworks have been of great interest due to their variable structural architectures and their subsequent properties [8] Alkaline earth metals are very reactive and show a wide range of coordination, two qualities that make them excellent candidates to construct a range of functional materials with specified structures and properties [9]. 


\section{Experimental}

\subsection{Reagents and Instrumentation}

All the chemical agents are commercially available and employed without further purification. The infrared spectra were recorded on a Shimadzu Transform IR, Shimadzu spectrometer in the range of 4000-400 $\mathrm{cm}^{-1}$. X-ray powder diffraction (XRD) measurements were performed by using a Bourevestnik Dron Poh-8 $(\mathrm{V}=40.00 \mathrm{Kv}$, Current $=20.08 \mathrm{~mA})$ diffractometer with monochromated $\mathrm{Cu}-\mathrm{k} \alpha$ radiation $(\lambda=1.54056 \AA$ ). A scanning electronmicroscope (SEM; TScan, VEGA, Czech Republic) was used to get micrographs of the MOF.

\subsection{Preparation of MOF}

To the best of our knowledge, this method of synthesis has not been previously reported and was used because it is an easy and low-risk method.

A mixture of $2 \mathrm{mmol} \mathrm{Ba}\left(\mathrm{NO}_{3}\right)_{2}(261.337 \mathrm{~g} / \mathrm{mol}), 0.2 \mathrm{mmol}$ benzene-1,2,4,5-tetracaboxylic acid 254.15 $\mathrm{g} / \mathrm{mol}$, and $150 \mathrm{~mL}$ of $\mathrm{CH}_{3} \mathrm{CN}: \mathrm{H}_{2} \mathrm{O}$ (v:v = 1:1) was placed in a completely isolated container in an ultrasonic bath for $6 \mathrm{~h}$. The temperature was slowly increased from room temperature to $80^{\circ} \mathrm{C}$. Finally, the formed MOF was cooled slowly, and the white precipitate was washed with deionized water.

According to a previous source, it was expected that a 3-dimensional 3D network would occur in the structure [10].

\section{Results and Discussion}

\subsection{X-ray Diffraction}

The phase purity of the MOF, found by powder XRD, is shown in Figure 1; the conformity of the main peaks in 2theta 10, 20, 25, 28, 30, 40, 45 and 50, between the title MOF and the simulated pattern, though with less intensity, indicates the formation of the title material. The differences in intensity may be attributed to the preferred orientation of the powder sample.

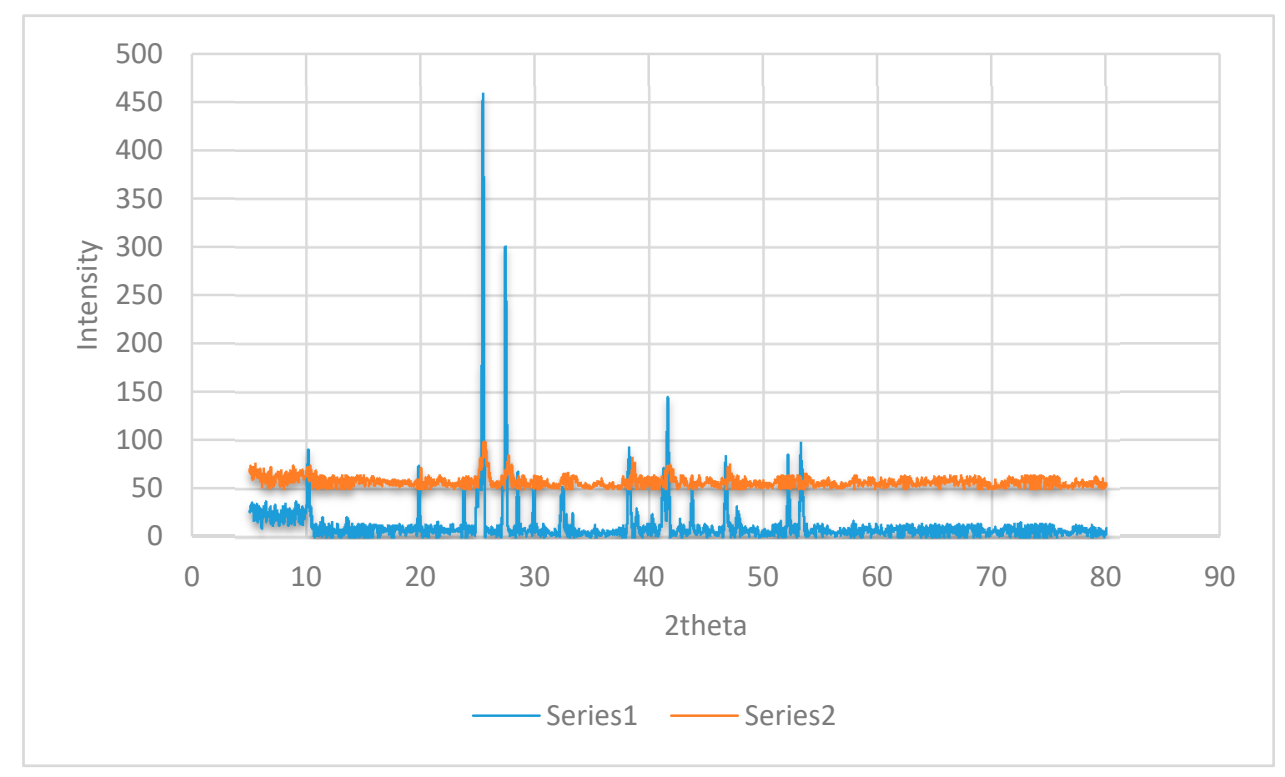

Figure 1. XRD Patterns for $\left\{\left[\mathrm{Ba}_{2}\left(\mathrm{H}_{2} \mathrm{btec}\right) \cdot \mathrm{H}_{2} \mathrm{O}\right] \cdot 0.5 \mathrm{H}_{2} \mathrm{O}\right\}_{\mathrm{n}}$ (Series 1: simulated; Series 2: experimental). 


\subsection{FTIR Spectra}

The FT-IR spectrum of the title MOF in the range of $4000-400 \mathrm{~cm}^{-1}$ was investigated, as shown in Figure 2. The strong peak of $3400 \mathrm{~cm}^{-1}$ is related to the $\mathrm{O}-\mathrm{H}$ stretching vibration of water molecules, and the peaks of $1690-1605$ and $690-900 \mathrm{~cm}^{-1}$ can be ascribed to asymmetric stretching vibrations of the carboxyl group and $\mathrm{C}-\mathrm{H}$ aromatic ring, respectively.

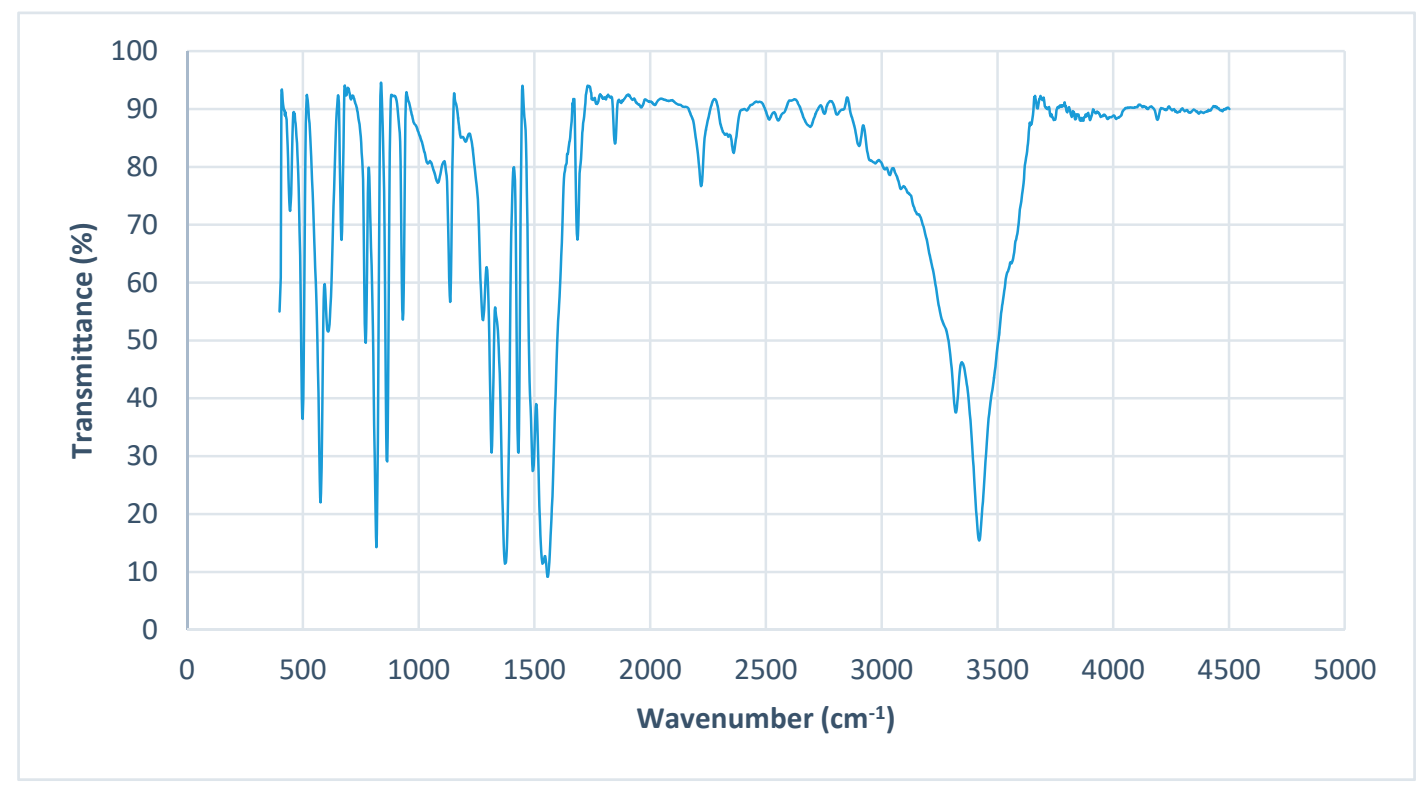

Figure 2. The FT-IR spectrum of the $\left\{\left[\mathrm{Ba}_{2}\left(\mathrm{H}_{2} \mathrm{btec}\right) \cdot \mathrm{H}_{2} \mathrm{O}\right] \cdot 0.5 \mathrm{H}_{2} \mathrm{O}\right\}_{\mathrm{n}}$ framework.

\subsection{SEM Analysis}

SEM gave excellent images of very small, rough particles of the material under investigation. Therefore, the SEM analysis, as seen in Figure 3, was used to identify the structural morphology of the material.

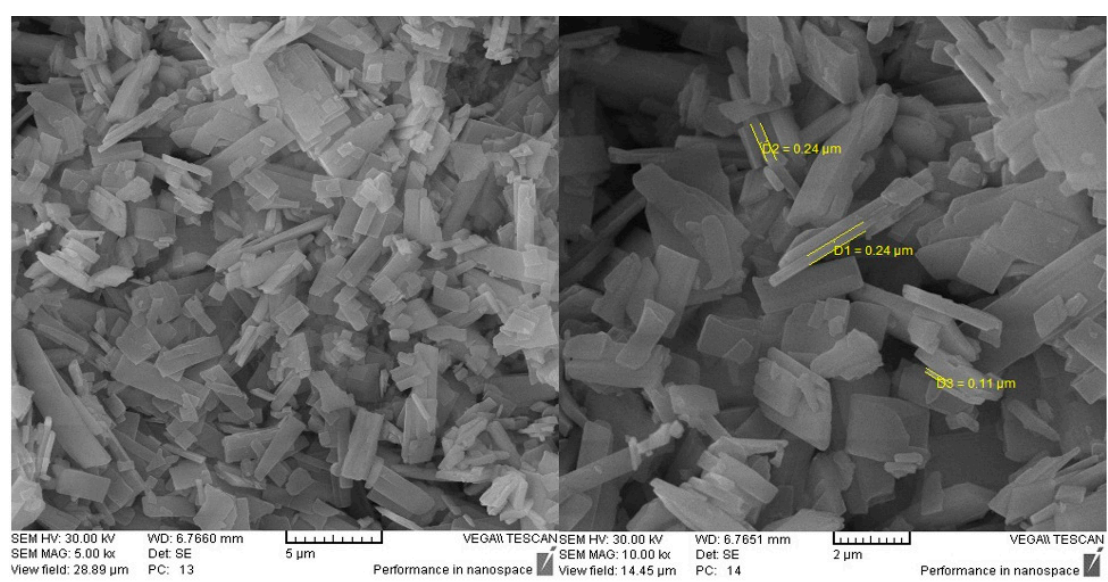

Figure 3. SEM images of the $\left\{\left[\mathrm{Ba}_{2}\left(\mathrm{H}_{2} \mathrm{btec}\right) \cdot \mathrm{H}_{2} \mathrm{O}\right] \cdot 0.5 \mathrm{H}_{2} \mathrm{O}\right\}_{\mathrm{n}}$ metal-organic framework

\section{Conclusions}

In summary, a three-dimensional framework based on Ba (II) and a benzene-1,2,4,5tetracaboxylic acid ligand was synthesized with a ultrasonic method. X-ray diffraction, Fourier transform infrared spectroscopy and scanning electron microscopy analyzes were used to identify this material. 


\section{Reference}

1. Rouhani, F.; Morsali, A. Frontispiece: Goal-Directed Design of Metal-Organic Frameworks for HgII and PbII Adsorption from Aqueous Solutions, Chem. A Eur. J. Chem. 2018, 24, 17170-17179.

2. Hou, Y.; Liu, L.; Zhang, Z.; Sun, J.; Zhang, Y.; Jiang, J.Z. Synthesis, crystal structures, and fluorescence properties of porphyrin alkaline earth MOFs, Inorg. Chem. Commun. 2018, 95, 36-39.

3. Cao, K.L.; Xia, Y.; Wang, G.X.; Feng, Y.L. A robust luminescent Ba (II) metal - organic framework based on pyridine carboxylate ligand for sensing of small molecules, Inorg. Chem. Commun. 2015, 53, 42-45.

4. Lin, Z.J.; Zheng, H.Q.; Zheng, H.Y.; Lin, L.P.; Xin, Q.; Cao, R. Efficient Capture and Effective Sensing of $\mathrm{Cr}_{2} \mathrm{O}_{7}{ }^{2-}$ from Water Using a Zirconium Metal-Organic Framework, Inorg. Chem. 2019, 56, 14178-14188.

5. Tahmasebi, E.; Masoomi, M.Y.; Yamini, Y.; Morsali, A. Application of Mechanosynthesized AzineDecorated Zinc(II) Metal-Organic Frameworks for Highly Efficient Removal and Extraction of Some Heavy-Metal Ions from Aqueous Samples: A Comparative Study, Inorg. Chem. 2015, 54, 425-433.

6. Vu, T.A.; Le, G.H.; Dao, C.D.; Dung, L.Q.; Nguyen, K.T.; Nguyen, Q.K.; Dang, P.T.; Tran, H.T.K.; Duong, Q.T.; Nguyen, T.V.; Lee, G.D. Arsenic removal from aqueous solutions by adsorption using novel MIL53(Fe) as a highly efficient adsorbent, RSC Adv. 2015, 5, 5261-5268.

7. Wang, Y.; Ye, G.; Chen, H.; Hu, X.; Niu, Z.; Ma, S. Functionalized metal-organic framework as a new platform for efficient and selective removal of cadmium (II) from aqueous solution, J. Mater. Chem. A. 2015, 3, 15292-15298.

8. Halake, S.; Ok, K.M. differential gas adsorption, high thermal stability, and reversible coordination of two new barium-organic frameworks, Ba(SBA)(DMF)4 and Ba2(BTEC)(H2O), J. Solid State Chem. 2015, 231, 132137.

9. Balendra, A.; Ramanan, A. Structural diversity of alkaline-earth 2,5-thiophenedicarboxylates, J. Mol. Struct. 2017, 1131, 171-180.

10. Du, S.; Ji, C.; Xin, X.; Zhuang, M.; Yu, X.; Lu, J.; Lu, Y.; Sun, D. Syntheses, structures and characteristics of four alkaline-earth metal-organic frameworks (MOFs) based on benzene-1,2,4,5-tetracarboxylicacid and its derivative ligand, J. Mol. Struct. 2017, 1130, 565-572.

(C) 2019 by the authors. Licensee MDPI, Basel, Switzerland. This article is an open access article distributed under the terms and conditions of the Creative Commons Attribution (CC BY) license (http://creativecommons.org/licenses/by/4.0/). 